\title{
Optimal radius of crystal curvature for planar channeling of high-energy negatively charged particles in a bent crystal
}

\author{
I. V. Kirillin* \\ Akhiezer Institute for Theoretical Physics of National Science Center \\ "Kharkov Institute of Physics and Technology", Akademicheskaya street 1, 61108 Kharkov, Ukraine \\ and V.N. Karazin Kharkov National University, Svobody square 4, 61022 Kharkov, Ukraine
}

(Received 28 July 2017; published 13 October 2017)

\begin{abstract}
The problem of planar channeling of high-energy negatively charged particles in a bent crystal was considered on the basis of analytical calculation and numerical simulation. We show the existence of an optimal radius of crystal curvature for planar channeling, corresponding to the maximum deflection of the particle beam. The analytical calculation was carried out in the parabolic planar potential approximation, and in the numerical simulation the Doyle-Turner approximation was used.
\end{abstract}

DOI: 10.1103/PhysRevAccelBeams.20.104401

\section{INTRODUCTION}

The use of a bent crystal to deflect high-energy charged particles was first proposed in Refs. [1,2]. It was suggested to use planar channeling [3] in a bent crystal for the deflection of particles. The assumption was that if charged particles move in a potential well formed by neighboring atomic planes of a bent crystal, these particles will deflect by following the bending of the crystalline atomic planes. Experimental confirmation of the proposed deflection mechanism for positively charged particles was obtained in Ref. [4]. For positively charged particles, the potential well formed by the neighboring atomic planes of the crystal, lies between the planes, while for the negatively charged particles the position of the center of potential well coincides with the position of the atomic plane. For this reason dechanneling caused by incoherent scattering of charged particles (which occurs primarily because of the thermal vibrations of the atoms of the crystals and scattering by the electron subsystem of atoms) is more intense for negatively charged particles than for positively charged ones. This is why planar channeling in a bent crystal is more effective for positively charged particles. Experimental confirmation of the possibility of using planar channeling in a bent crystal to deflect negatively charged particles was obtained in Ref. [5]. The deflection efficiency in that experiment was approximately $30 \%$. In recent years, a number of experiments have been carried out to study the planar channeling of negatively charged particles in bent crystals $[6,7]$. These experiments demonstrated the deflection of a part of the electron beam in different energy

*kirillin@kipt.kharkov.ua

Published by the American Physical Society under the terms of the Creative Commons Attribution 4.0 International license. Further distribution of this work must maintain attribution to the author(s) and the published article's title, journal citation, and DOI. ranges of particles from $3.35 \mathrm{GeV}$ to $14 \mathrm{GeV}$ at fixed crystal parameters. In this paper, we carry out a theoretical analysis of planar channeling of negatively charged particles in a bent crystal and discuss the optimal crystal parameters for observing the deflection of negatively charged particles.

\section{OPTIMAL RADIUS OF CURVATURE}

It was recently shown in [8] that if a bent crystal is oriented axially with respect to the initial momentum of high-energy negatively-charged particles there is an optimal radius of crystal curvature corresponding to the deflection of the particle beam by the maximum angle. The existence of the optimal radius of curvature is explained by the fact that at small radii of curvature of the crystal the particles quickly leave the deflection mode because of the strong centrifugal force (which is inversely proportional to the radius of curvature), and at large radii of curvature, it becomes necessary to increase the thickness of the crystal (since the bending angle of the crystals is equal to the ratio of its thickness to the radius of curvature), which leads to the escape of particles from the deflection mode due to incoherent scattering (the mean square of the incoherent scattering angle is proportional to the crystal thickness). In the case of planar channeling of negatively charged particles in a bent crystal, both of these factors (centrifugal force and incoherent scattering) also lead to the escape of particles from the deflection mode. Thus, one should expect the presence of optimal radius of curvature in the case of planar channeling of negatively charged particles in a bent crystal. It should be noted that the existence of the optimal radius of crystal curvature for positively charged particles deflection had been shown for the first time in [9] and the analytical expression for the optimal crystal curvature for positively charged particles was suggested in $[10,11]$.

In a straight crystal the equation of particle motion for planar channeling can be written as follows [12] 


$$
\frac{d^{2} x}{d t^{2}}=-\frac{c^{2}}{E} \frac{d U_{\mathrm{p}}(x)}{d x}
$$

where the $x$-axis is orthogonal to the planes forming the channel, $U_{\mathrm{p}}(x)$ is the potential energy of the particle in the field of crystal atomic planes, $E$ is the energy of the particle. If the crystal is bent, in a noninertial frame of reference connected with a bent crystalline plane, the trajectory of the particle can be found by solving the Eq. (1), in which the term $-\frac{c^{2}}{R}$ is added to the right side. This additional term appears due to the presence of centrifugal force $-\frac{E}{R}$. Thus, the equation of motion of particles for planar channeling in a bent crystal can be written as

$$
\frac{d^{2} x}{d t^{2}}=-\frac{c^{2}}{E} \frac{d U_{\mathrm{eff}}(x)}{d x}
$$

where $U_{\text {eff }}(x)=U_{\mathrm{p}}(x)+E x / R$ is an effective potential energy of the particle.

For simplicity of calculation we will use the parabolic approximation of planar potential

$$
\begin{aligned}
U_{\mathrm{p}}(x)= & -\frac{U_{0}}{d_{\mathrm{p}}^{2}}\left\{\left(2 x+d_{\mathrm{p}}\right)^{2} H\left[-x\left(d_{\mathrm{p}}+x\right)\right]\right. \\
& \left.+\left(2 x-d_{\mathrm{p}}\right)^{2} H\left[x\left(d_{\mathrm{p}}-x\right)\right]\right\},
\end{aligned}
$$

where $d_{\mathrm{p}}$ is the distance between neighboring atomic planes and $H(x)$ is the Heaviside step function which equals to 1 for positive arguments and to 0 for negative ones. In [7] it was shown that such a simple parabolic approximation gives almost the same result for estimating the fraction of negatively charged particles that becomes under-barrier when the beam impinges on a crystal, as a more complex and realistic Doyle-Turner approximation [13]. In Fig. 1 we showed the comparison between potential energy in the parabolic approximation (3) (red dashed line) and potential energy in the field of atomic planes in the Doyle-Turner approximation (black solid line) for Si (110) planes for a particle with a charge that equals to the charge of an electron.

Let the beam of high-energy negatively charged particles impinges on a bent crystal and the initial momentum of each of the particles in the beam lies in the plane that form the planar channel. To find the fraction of negatively charged particles that becomes under-barrier when the beam impinges on the bent crystal one must first find negative $x$ for which the derivative of the effective potential equals to zero. If the planar potential is chosen in the parabolic form (3), this point can be found as $x_{\text {neg }}=\frac{d_{\mathrm{p}}}{2}\left(\frac{R_{c}}{R}-1\right)$, where $R_{\mathrm{c}}=\frac{E d_{\mathrm{p}}}{4 U_{0}}$ is the critical radius of planar channeling in the parabolic approximation of the potential. Further, the positive $x$ for which $U_{\text {eff }}(x)=U_{\text {eff }}\left(x_{\text {neg }}\right)$ can be found as $x_{\mathrm{pos}}=\frac{d_{\mathrm{p}}}{2}\left(\frac{R_{c}}{R}+1-2 \sqrt{\frac{R_{\mathrm{c}}}{R}}\right)$. Now if $R>R_{\mathrm{c}}$ one can find

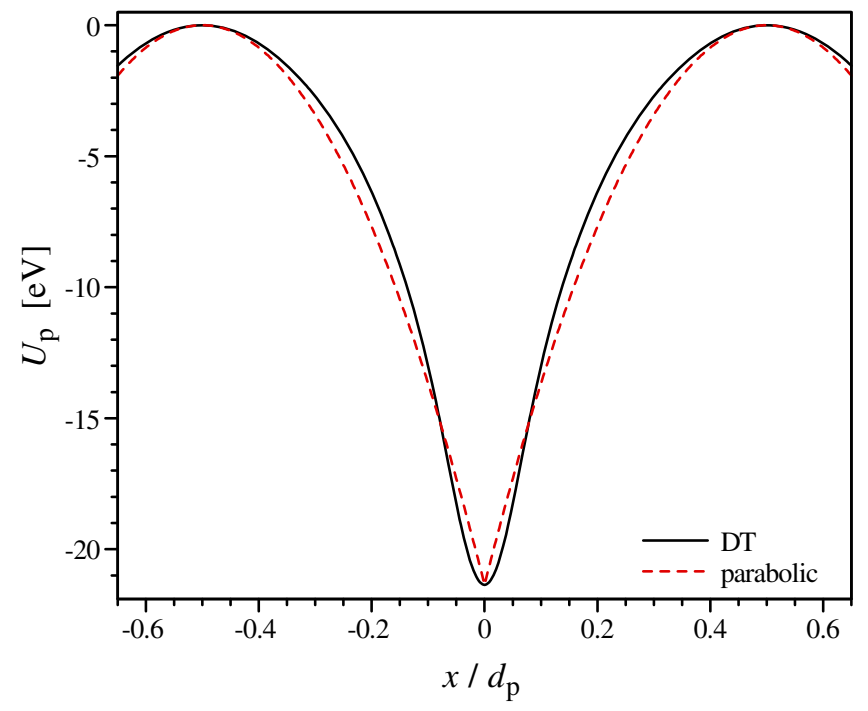

FIG. 1. The potential energy of a particle with a charge, that equals to the charge of an electron, in the field of (110) atomic planes of Si. Black solid line corresponds to the Doyle-Turner approximation (DT), red dashed line corresponds to the parabolic approximation (3).

the fraction of under-barrier negatively charged particles in the moment when the beam impinges on a bent crystal as $\frac{x_{\mathrm{pos}}-x_{\text {neg }}}{d_{\mathrm{p}}}=1-\sqrt{\frac{R_{\mathrm{c}}}{R}}$. If $R<R_{\mathrm{c}}$, there are no under-barrier particles.

During the motion of particles in a bent crystal, some particles become over-barrier due to incoherent multiple scattering caused by thermal vibrations of atoms and scattering by atomic electrons. In amorphous medium, the angular distribution of particles due to multiple scattering is Gaussian [14]. By analogy, we assume that the angular distribution due to incoherent multiple scattering in a crystal also is Gaussian. If so, for $R>R_{c}$ on some crystal thickness $l$ the fraction of under-barrier (channeled) particles $f$ can be estimated as

$$
\begin{aligned}
f & =\left(1-\sqrt{\frac{R_{\mathrm{c}}}{R}}\right) \frac{1}{\sqrt{2 \pi} \theta_{0}} \int_{-\theta_{\mathrm{c}}}^{\theta_{\mathrm{c}}} \exp \left(-\frac{\theta^{2}}{2 \theta_{0}^{2}}\right) d \theta \\
& =\left(1-\sqrt{\frac{R_{\mathrm{c}}}{R}}\right) \operatorname{erf}\left(\frac{\theta_{c}}{\sqrt{2} \theta_{0}}\right),
\end{aligned}
$$

where $\theta$ is the angle between the crystal planes and momentum of the particle, $\theta_{0}$ is the standard deviation of the angular distribution and $\theta_{\mathrm{c}}$ is the critical angle for planar channeling. The critical angle for planar channeling in a bent crystal in the parabolic approximation of planar potential can be found as $\theta_{\mathrm{c}}=\sqrt{\frac{2 U_{0}}{E}}\left(1-\frac{R_{c}}{R}\right)$ [11].

In oriented crystal the angular distribution caused by incoherent scattering depends on the transverse energy of channeled particles. However, for simplicity of consideration 
let us assume that by analogy with multiple scattering in amorphous medium $\theta_{0} \propto \sqrt{l}$ and $\xi$ is the coefficient of proportionality. In this approximation from Eq. (4) one can find that

$$
\theta_{0}=\frac{\theta_{\mathrm{c}}}{\sqrt{2} \operatorname{erf}^{-1}\left(\frac{f}{1-\sqrt{\frac{R_{\mathrm{c}}}{R}}}\right)}=\xi \sqrt{l},
$$

where $\operatorname{erf}^{-1}(x)$ is the inverse error function.

From Eq. (5), one can find the thickness of the crystal $l$ on which the fraction of channeled particles equals to $f$ :

$$
l=\frac{\theta_{\mathrm{c}}^{2}}{2 \xi^{2}\left[\operatorname{erf}^{-1}\left(\frac{f}{1-\sqrt{\frac{R_{\mathrm{c}}}{R}}}\right)\right]^{2}} .
$$

Then, knowing that the bending angle of the crystal equals to the crystal thickness divided by the radius of curvature, one can find the deflection angle $\alpha$ up to which the fraction of channeled particles is higher than $f$ :

$$
\alpha=\frac{l}{R}=\frac{\theta_{\mathrm{c}}^{2}}{2 \xi^{2} R\left[\operatorname{erf}^{-1}\left(\frac{f}{1-\sqrt{\frac{R_{\mathrm{c}}}{R}}}\right)\right]^{2}} .
$$

The shape of the dependence (7) is shown in Fig. 2. This figure is plotted without vertical scale just to show the existence of the maximum in the dependence of $\alpha$ on the radius of curvature.

The optimal radius of curvature which corresponds to the maximum $\alpha$ can be found by solving the equation

$$
\left.\frac{d \alpha}{d R}\right|_{R=R_{\mathrm{opt}}}=0 .
$$

It should be noted that according to Eq. (7) the ratio $R_{\text {opt }} / R_{\mathrm{c}}$ does not depend on particle energy, but only on the

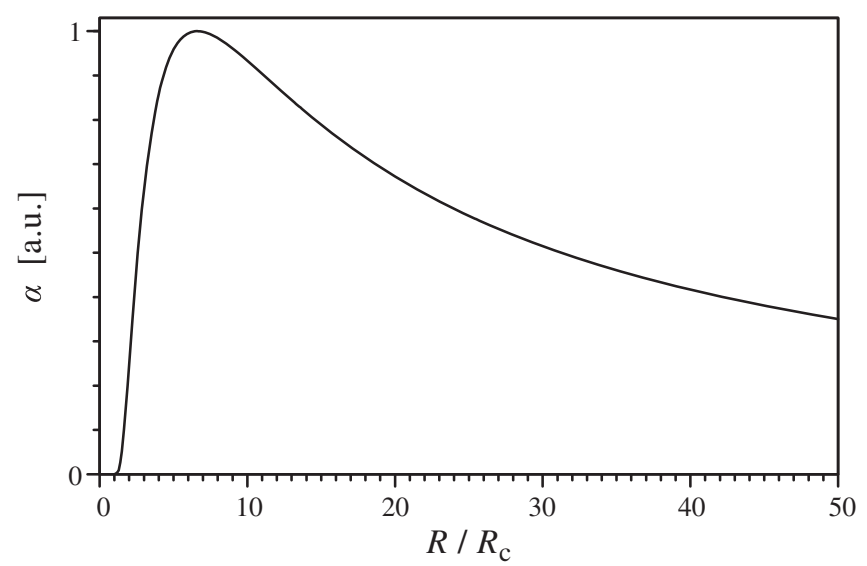

FIG. 2. The shape of the dependence of the deflection angle up to which the fraction of channeled particles is higher than $f$ on the radius of curvature.

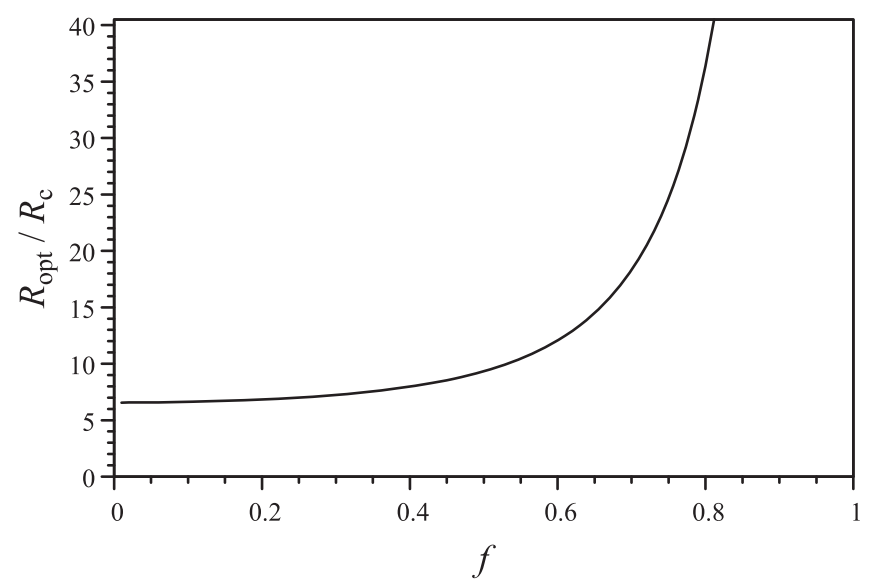

FIG. 3. The dependence of the ratio of the optimal radius of curvature to the critical radius of planar channeling on the fraction of channeled particles in the parabolic approximation of planar potential.

value of $f$. The dependence of $R_{\mathrm{opt}} / R_{\mathrm{c}}$ on $f$ in the parabolic approximation of planar potential is shown in Fig. 3. We see that for a wide range of $f \frac{R_{\text {opt }}}{R_{\mathrm{c}}}$ takes values from 6.5 to 7 .

\section{SIMULATION}

Now we will find the dependence of $\alpha$ on $R / R_{\mathrm{c}}$ in more realistic Doyle-Turner approximation of planar potential. In this approximation the potential energy of a particle with a charge, that equals to the charge of an electron, in the field of an atomic string could be written as

$$
U_{\mathrm{str}}(\rho)=-\frac{8 \pi^{2} \hbar^{2}}{m_{\mathrm{e}} d} \sum_{k=1}^{4} \frac{\alpha_{k}}{\beta_{k}+B} e^{-\frac{4 \pi^{2} \rho^{2}}{\beta_{k}+B}}
$$

where $m_{\mathrm{e}}$ is an electron mass, $d$ is the distance between neighboring atoms in the atomic string, $\alpha_{k}$ and $\beta_{k}$ are coefficients found in [13] for a large number of elements, $B=8 \pi^{2}\left\langle r_{T}{ }^{2}\right\rangle$ and $r_{T}$ is the rms atomic thermal vibration amplitude in one direction $\left(r_{T} \approx 0.075 \AA\right.$ for $\mathrm{Si}$ at $\left.293 \mathrm{~K}\right)$, $\rho=\sqrt{x^{2}+y^{2}}$ is the distance from the atomic string. After averaging of (9) over $y$ one can obtain the potential energy of a particle with a charge, that equals to the charge of an electron, in the field of an atomic plane as

$$
U_{\mathrm{pl}}(x)=-\frac{4 \pi^{\frac{3}{2}} \hbar^{2}}{m_{\mathrm{e}} d d_{\mathrm{s}}} \sum_{k=1}^{4} \frac{\alpha_{k}}{\sqrt{\beta_{k}+B}} e^{-\frac{4 \pi^{2} x^{2}}{\beta_{k}+B}},
$$

where $d_{\mathrm{s}}$ is the distance between neighboring atomic strings in the atomic plane.

To obtain the potential of atomic planes in a crystal in the Doyle-Turner approximation one must sum up potentials of atomic planes (10). Since $U_{\mathrm{pl}}(x)$ in Eq. (10) decreases rapidly with increasing distance from the atomic plane, only a limited number of neighboring atomic planes 
determine the value of the potential at the selected point inside the crystal. This fact gives us a possibility to sum the potentials of atomic planes analytically and find the potential energy of a high-energy charged particle with a charge that equals to the charge of an electron as

$$
U_{\mathrm{p}}(x)=\sum_{n=-\infty}^{\infty} U_{\mathrm{pl}}\left(x-x_{n}\right),
$$

where points $x_{n}$ correspond to the location of atomic planes. After summation one can obtain

$$
U_{\mathrm{p}}(x)=-\frac{2 \pi \hbar^{2}}{m_{\mathrm{e}} d d_{\mathrm{s}} d_{\mathrm{p}}} \sum_{k=1}^{4} \alpha_{k} \theta_{3}\left(\pi \frac{x}{d_{p}}, e^{-\frac{\beta_{k}+B}{4 d_{\mathrm{p}}^{2}}}\right),
$$

where $\theta_{3}(u, q)=\sum_{n=-\infty}^{\infty} q^{n^{2}} e^{2 n u i}$ is the Jacobi theta function of the third kind [15], $i^{2}=-1$.

Unfortunately, the expression (12) is too complex to obtain the dependence of $\alpha$ on $R / R_{\mathrm{c}}$ analytically, as we did in the previous section for parabolic potential. However, this dependence could be found with a help of numerical simulation. We carried out a numerical simulation of $\pi^{-}$-mesons motion in a bent $\mathrm{Si}$ crystal. The energies of particles were $10 \mathrm{GeV}, 100 \mathrm{GeV}$, and $1 \mathrm{TeV}$. The simulation code was the same as in Refs. [16,17]. The code computes the trajectory of a high-energy charged particle in the field of continuous atomic string potential (in the Doyle-Turner approximation) through numerical integration of the equation of motion. It takes into account the incoherent scattering caused by thermal vibrations of atoms and scattering on electrons. Other kinds of incoherent scattering were not taken into account considering the small crystal thickness.

In Fig. 4 the orientation of the crystal toward the impinging beam is shown. The angle $\psi$ between the initial momentum of each particle $\vec{p}$ and $(x, z)$ plane significantly exceeded the critical angle of axial channeling $\psi_{\mathrm{c}}$ [3] $\left(\psi>50 \psi_{\mathrm{c}}\right)$. The initial value of $p_{x}$ in simulation was equal to zero. The bending plane was $(x, z)$ plane.

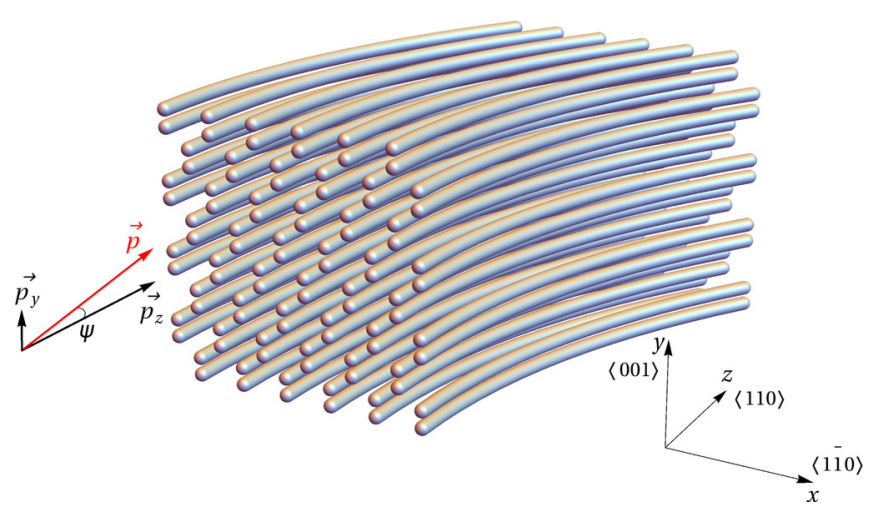

FIG. 4. The orientation of Si crystal towards the impinging beam of $\pi^{-}$-mesons.

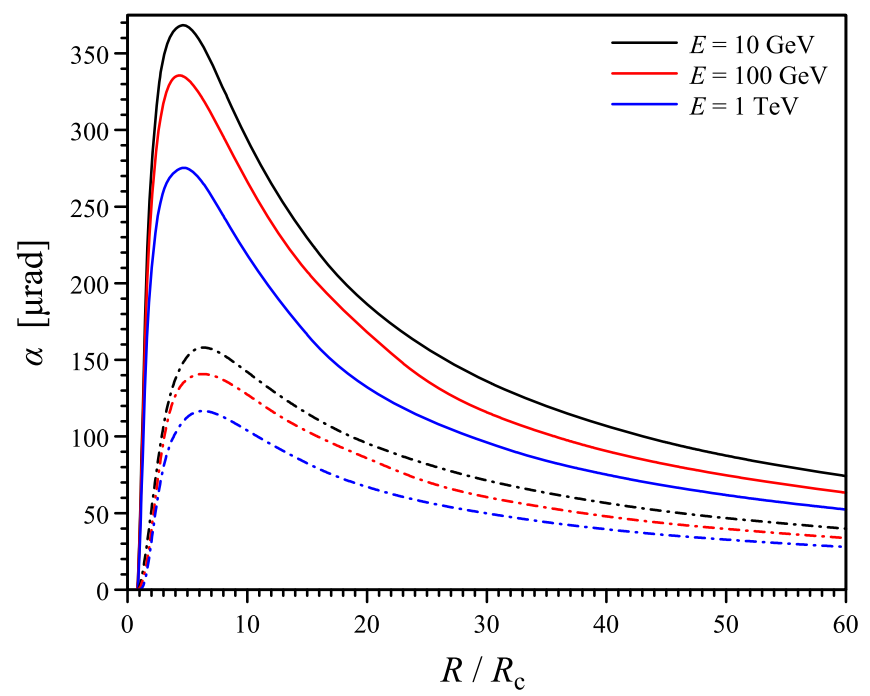

FIG. 5. The dependence of the deflection angle up to which the fraction of channeled particles is higher than 5\% (solid lines) and $15 \%$ (dash-dotted lines) on the ratio of radius of curvature to the critical radius of planar channeling.

The results of simulation are shown in Fig. 5. Solid lines correspond to the dependence of $\alpha$ on $R / R_{\mathrm{c}}$ for $f=5 \%$, while dash-dotted lines correspond to $f=15 \%$. The black lines correspond to $E=10 \mathrm{GeV}$, the red lines to $E=$ $100 \mathrm{GeV}$ and the blue lines to $E=1 \mathrm{TeV}$. The shape of the dependences of $\alpha$ on $R / R_{\mathrm{c}}$ in Fig. 5 is similar to the shape of the dependence that is shown in Fig. 2. It is well seen that like in the parabolic approximation of planar potential the ratio $R_{\text {opt }} / R_{\mathrm{c}}$ almost does not depend on particle energy, but only on the value of $f$. From simulation we obtained for $f=5 \%$ that $R_{\text {opt }} / R_{\mathrm{c}} \approx 4.5$ and for $f=15 \%$ that $R_{\text {opt }} / R_{\mathrm{c}} \approx 6.2$. These values are somewhat lower than those obtained in the parabolic approximation of the potential. It should be noted that despite the intensity of incoherent scattering decreases with the increase of particle energy, the maximum value of $\alpha$ decreases with the increase of particle energy due to the decrease of the critical angle of planar channeling. It should also be noted that, despite the fact that the value of the optimal radius of curvature varies little with a change in $f$ from $5 \%$ to $15 \%$, the maximum value of $\alpha$ decreases very significantly at the same time.

\section{CONCLUSIONS}

In both used approximations of planar potential of the crystal it was shown that the dependence of the deflection angle, up to which the fraction of channeled particles is higher than some certain value $f$, has a maximum. The existence of this maximum means the existence of the optimal radius of curvature. However, in contrast to the case of stochastic deflection [8] the value of the optimal radius of curvature depends on $f$, because after the particle beam impinges on the crystal not all particles become 
under-barrier (channeled). The found dependence of the ratio of the optimal radius of curvature to the critical radius of planar channeling on the fraction of channeled particles may be used for obtaining optimal conditions for highenergy negatively charged particle beam deflection by means of planar channeling in a bent crystal. Such optimization can be exploited for beam manipulation as on existing accelerators of negatively charged particles, and on future muon or electron-positron colliders.

\section{ACKNOWLEDGMENTS}

This work was supported by the President's of Ukraine Grant No. F70/110-2017 of the State Fund for Fundamental Research, the National Academy of Sciences of Ukraine (projects CO-1-8/2017 and F56-2017) and the Ministry of Education and Science of Ukraine (project no. $0117 \mathrm{U} 004866)$.

[1] E. N. Tsyganov, Fermilab Report No. TM-682, 1976.

[2] E. N. Tsyganov, Fermilab Report No. TM-684, 1976.

[3] J. Lindhard, Influence of crystal lattice on motion of energetic charged particles, Danske Vid. Selsk. Mat. Fys. Medd. 34, 14 (1965).

[4] A. F. Elishev et al., Steering of charged particle trajectories by a bent crystal, Phys. Lett. B 88, 387 (1979).

[5] W. Scandale et al., Observation of channeling and volume reflection in bent crystals for high-energy negative particles, Phys. Lett. B 681, 233 (2009).

[6] U. Wienands, T. W. Markiewicz, J. Nelson, R. J. Noble, J. L. Turner, U. I. Uggerhøj, T. N. Wistisen, E. Bagli, L. Bandiera, G. Germogli, V. Guidi, A. Mazzolari, R. Holtzapple, and M. Miller, Observation of Deflection of a Beam of Multi-GeV Electrons by a Thin Crystal, Phys. Rev. Lett. 114, 074801 (2015).

[7] T. N. Wistisen, U. I. Uggerhøj, U. Wienands, T. W. Markiewicz, R. J. Noble, B. C. Benson, T. Smith, E. Bagli,
L. Bandiera, G. Germogli, V. Guidi, A. Mazzolari, R. Holtzapple, and S. Tucker, Channeling, volume reflection, and volume capture study of electrons in a bent silicon crystal, Phys. Rev. Accel. Beams 19, 071001 (2016).

[8] I. V. Kirillin, N. F. Shul'ga, L. Bandiera, V. Guidi, and A. Mazzolari, Influence of incoherent scattering on stochastic deflection of high-energy negative particle beams in bent crystals, Eur. Phys. J. C 77, 117 (2017).

[9] A. M. Taratin, Yu. M Filimonov, E. G. Vyatkin, and S. A. Vorobiev, Theory of planar channeling of relativistic protons in bent crystals, Phys. Status Solidi B 100, 273 (1980).

[10] M. D. Bavizhev, V. M. Biryukov, and Y. G. Gavrilov, Efficiency of steering of a beam of high energy protons by an optimally bent crystal. Influence of temperature on the efficiency, Sov. Phys. Tech. Phys. 36, 203 (1991).

[11] V. M. Biryukov, Y. A. Chesnokov, and V. I. Kotov, Crystal Channeling and Its Application at High-Energy Accelerators (Springer, Berlin, 1997).

[12] A. I. Akhiezer and N. F. Shul'ga, High-Energy Electrodynamics in Matter (Gordon and Breach Publ., Amsterdam, 1996).

[13] P. A. Doyle and P. S. Turner, Relativistic Hartree-Fock X-ray and electron scattering factors, Acta Crystallogr. Sect. B 24, 390 (1968).

[14] V. L. Highland, Some practical remarks on multiple scattering, Nucl. Instrum. Methods 129, 497 (1975).

[15] I. S. Gradshteyn and I. M. Ryzhik, Table of Integrals, Series, and Products, 7th ed. (Academic Press, London, 2007).

[16] N. F. Shul'ga, I. V. Kirillin, and V. I. Truten', Dynamical chaos and stochastic mechanism of high-energy negatively charged particle deflection by bent crystals, Phys. Lett. B 702, 100 (2011).

[17] N. F. Shul'ga, I. V. Kirillin, and V. I. Truten, Stochastic mechanism of a high-energy charged-particle beam deflection by a bent crystal, Nuovo Cimento Soc. Ital. Fis. C 34, 425 (2011). 